\title{
SEMICLASSICAL ANALYSIS OF A NONLINEAR EIGENVALUE PROBLEM AND NONANALYTIC HYPOELLIPTICITY
}

\author{
BERNARD HELFFER, DIDIER ROBERT, AND XUE PING WANG
}

Dedicated to M. Sh. Birman on the occasion of his 75th birthday

\begin{abstract}
A semiclassical analysis of a nonlinear eigenvalue problem arising from the study of the failure of analytic hypoellipticity is given. A general family of hypoelliptic, but not analytic hypoelliptic operators is obtained.
\end{abstract}

\section{$\S 1$. INTRODUCTION}

We are interested in a family of operators of the type

$$
H\left(x, D_{x}, \lambda\right)=-\Delta+(P(x)-\lambda)^{2},
$$

where $x \mapsto P(x)$ is a polynomial of degree $m$. In the study of the failure of analytic hypoellipticity, one approach consists in showing that the nonlinear eigenvalue problem

$$
H\left(x, D_{x}, \lambda\right) v=0
$$

has at least one nontrivial solution $(\lambda, v) \in \mathbb{C} \times \mathcal{S}\left(\mathbb{R}^{n}\right)(v \neq 0)$, where $\mathcal{S}\left(\mathbb{R}^{n}\right)$ is the Schwartz space. This has been used by many authors including Baouendi-Goulaouic, Helffer, Christ [3, 4, 5, 6], Hanges-Himonas [9], Chanillo [1], and quite recently, ChanilloHelffer-Laptev [2], where the reader can find a more extensive list of references. All the results lead to the formulation of a conjecture by Trèves 23] giving a necessary and sufficient condition of analytic hypoellipticity that extends [14]. A rather natural conjecture is as follows: if $x \mapsto P(x)$ is a homogeneous elliptic polynomial on $\mathbb{R}^{n}$ of order $m>1$, then (1.2) has at least one nontrivial solution. This result was proved in [3, 17] for $n=1$, and in [2, for $m \geq 2 n$ and $n=2,3$. Our aim is to provide a semiclassical approach to this problem. Actually, our analysis concerns a class of operators of the form

$$
\sum_{j=1}^{p} D_{x_{j}}^{2}+\left(P\left(x_{1}, \ldots, x_{p}\right) D_{x_{p+1}}-D_{x_{p+2}}\right)^{2}+\left(Q\left(x_{1}, \ldots, x_{p}\right) D_{x_{p+1}}\right)^{2} .
$$

The analysis of analytic hypoellipticity for these operators can be reduced to a nonlinear eigenvalue problem for the operator $-\Delta_{x}+(P(x)-\lambda)^{2}+(Q(x))^{2}$. Specializing to the case of (1.1) $(Q=0)$, we obtain the following statement.

Theorem 1.1. Let $n$ be even. Let $P$ be any real polynomial of degree $m \geq 2$ such that its homogeneous part of degree $m$ is elliptic. Then the nonlinear eigenvalue problem (1.2) has at least one nontrivial solution.

2000 Mathematics Subject Classification. Primary 35P30, 35P25.

Key words and phrases. Semiclassical analysis, analytic hypoellipticity, nonlinear eigenvalue problem.

The first author was partially supported by the SPECT ESF European program. The third author was partially supported by the program "Outstanding Overseas Chinese Scholars" of the Chinese Academy of Sciences. 

[2]).

We formulate a standard consequence concerning analytic hypoellipticity (see, e.g.,

Corollary 1.2. Let $P$ be elliptic homogeneous of degree $m \geq 2$ on $\mathbb{R}^{p}$ with an even $p>0$. Then the operator on $\mathbb{R}^{p+2}$ given by the formula

$$
H\left(x, D_{x}\right):=\sum_{j=1}^{p} D_{x_{j}}^{2}+\left(P\left(x_{1}, \ldots, x_{p}\right) D_{x_{p+1}}-D_{x_{p+2}}\right)^{2}
$$

is not analytic hypoelliptic at 0.

Remark 1.3. As will be discussed more deeply in 95 , G. Métivier already showed in 13 that $H\left(x, D_{x}\right)$ is not analytic hypoelliptic on any open set. Actually, this operator is a sum of squares with an odd number of linearly independent vector fields. But the corollary presented here and its proof give a stronger information at 0 .

Other examples in any dimension, related to (1.3), are also given in 94 These examples shed a new light to the general conjecture of [23].

Recently, Chanillo-Helffer-Laptev [2] used Lidskiil's theorem to prove the existence of nonlinear eigenvalues of (1.2), which made it possible to recover some known results for $n=1$ and to give new examples in dimension $n \geq 2$.

The proof of Theorem 1.1 is based on the semiclassical analysis combined with the ideas of Chanillo-Helffer-Laptev [2]. We follow closely the reduction of Chanillo-HelfferLaptev, which is recalled in \$2. In this approach via Lidskiı's theorem, the existence of a nonlinear eigenvalue of (1.2) reduces to proving that the trace of the $k$ th power of a certain linear operator $\mathcal{D}$ does not vanish for some $k$ :

$$
\operatorname{Tr} \mathcal{D}^{k} \neq 0 \text {. }
$$

Our approach is to introduce a semiclassical parameter $h$ in this operator artificially (see (33). Then, the existence of a nonlinear eigenvalue of (1.2) is reduced to the proof of the relation

$$
\operatorname{Tr} \mathcal{D}(h)^{k} \neq 0
$$

for some $k$ and $h$, where $\mathcal{D}(h)$ is an $h$-pseudodifferential operator. In other words, we can say that, while Chanillo, Helffer, and Laptev tried to prove the relation

$$
\sum_{j} \lambda_{j}^{-k} \neq 0
$$

for some $k$, where the $\lambda_{j}$ are the nonlinear eigenvalues of (1.2) (which cannot be real under our assumptions), we want to find $h$ and $k$ such that

$$
\sum_{j}\left(h^{m /(m+1)} \lambda_{j}+1\right)^{-k} \neq 0 .
$$

It is standard (see [18]) that $\operatorname{Tr} \mathcal{D}(h)^{k}$ has a complete semiclassical expansion as $h \rightarrow 0$, which is computable theoretically. Now, the question amounts to finding a nonzero term in these expansions. We call this the semiclassical criterion. The computation of the leading term given in $\$$ yields the complete answer for $n$ even. Although we do not have a general result for all $n$ odd, we believe that the semiclassical criterion presented below can be used to show that, for each odd value of $n$, (1.2) has at least one nontrivial solution. The introduction of a semiclassical parameter $h$ permits us to overcome the difficulty related to the noncommutativity of operators encountered in [2]. 
Acknowledgements. The authors would like to thank S. Chanillo, M. Christ, A. Laptev, and F. Trèves for useful discussions, comments, or correspondence. Also, we thank J. Sjöstrand for informing us about the reference [16], and the referee for his or her careful reading. This work was completed when two of the authors (B. H. and X. P. W.) were invited at the MSRI in Berkeley.

\section{§2. Chanillo-Helffer-Laptev's approach}

We rewrite $H\left(x, D_{x}, \lambda\right)$ in the form

$$
H\left(x, D_{x}, \lambda\right)=L-2 \lambda M+\lambda^{2}
$$

$$
L=-\Delta+P(x)^{2}, \quad M=P(x) .
$$

The operator $L$ is invertible, and its inverse is a pseudodifferential operator (see [18]). It is also easy to give sufficient conditions for determining whether the operator

$$
A:=L^{-1}
$$

belongs to a given Schatten class (see the Appendix in [2]). Then the initial problem is reduced to the spectral analysis of the equation

$$
\left(I-2 \lambda B+\lambda^{2} A\right) u=0
$$

with

$$
B=A^{1 / 2} P A^{1 / 2} .
$$

In [2, Chanillo, Helffer, and Laptev used a rather standard approach to transform this nonlinear spectral problem to a linear one, and then applied Lidskin's theorem to prove the existence of a nontrivial solution.

First, we recall the reduction to the linear spectral problem. It is easily seen that it suffices to show that the operator $\mathcal{D}$ defined by

$$
\mathcal{D}:=\left(\begin{array}{cc}
2 B & A^{1 / 2} \\
-A^{1 / 2} & 0
\end{array}\right)
$$

has a nonzero eigenvalue $\mu$. The first component $u$ of the corresponding eigenvector is an eigenvector of problem (2.4) with $\lambda=\frac{1}{\mu}$ and, for $v=A^{\frac{1}{2}} u$, the pair $(\lambda, v)$ is a nontrivial solution of 1.2 .

If $B$ and $A$ are compact, then $\mathcal{D}$ is also compact, but the main difficulty is that $\mathcal{D}$ is not selfadjoint. We invoke the Lidskil theorem.

Theorem 2.1. Let $\mathcal{C}$ be a trace class operator. Let $\lambda_{j}(\mathcal{C})$ denote the sequence of nonzero eigenvalues of $\mathcal{C}$. Then

$$
\sum_{j} \lambda_{j}(\mathcal{C})=\operatorname{Tr} \mathcal{C}
$$

Here the eigenvalues are repeated in accordance with their algebraic multiplicity.

By Lidskilı's theorem, if $\operatorname{Tr} \mathcal{C} \neq 0$, then $\mathcal{C}$ has at least one nonzero eigenvalue.

Corollary 2.2 (Chanillo-Helffer-Laptev [2]). Let $\mathcal{D}$ be a compact operator. Assume that there exists $k \in \mathbb{N}^{*}(\equiv \mathbb{N} \backslash\{0\})$ such that $\mathcal{D}^{k}$ is of trace class and $\operatorname{Tr} \mathcal{D}^{k} \neq 0$. Then (1.2) has at least one nontrivial solution.

Chanillo, Helffer, and Laptev used this criterion in the case of $k=2,3,4$ and obtained a family of interesting results about nonanalytic hypoellipticity. The noncommutativity between $A$ and $B$ makes it hard to apply their method for larger values of $k$ (although some results can be obtained in the same vein for $k=6,8$ ). 


\section{§3. The SEMIClassical CRITERION}

As was explained in the Introduction, our goal is to incorporate a semiclassical parameter $h$ in the operators $A$ and $B$ and to apply the theory of $h$-pseudodifferential operators in order to give a complete semiclassical asymptotic expansion of the trace of $\mathcal{D}(h)^{k}$. Then we must find conditions under which the leading term is nonzero.

Initially, the family of operators to study looks like this:

$$
H\left(x, D_{x}, \lambda\right)=-\Delta+(P(x)-\lambda)^{2},
$$

where $x \mapsto P(x)$ is a real polynomial on $\mathbb{R}^{n}$ of order $m \geq 2$. We write $P$ in the form

$$
P=P_{m}+P_{m-1}+\cdots+P_{0},
$$

where $P_{j}$ is a homogeneous polynomial of degree $j$. We assume that $P_{m}$ is elliptic on $\mathbb{R}^{n}$ :

$$
P_{m}(x) \neq 0, \quad x \neq 0 .
$$

For definiteness, we assume that $P_{m}(x)>0, x \neq 0$. The case where $P_{m}(x)<0, x \neq 0$, is similar. This condition on $P_{m}$ requires $m$ to be even and, thus, excludes polynomials of odd degree for $n=1$.

To introduce the semiclassical parameter $h$, we apply the dilation $x \rightarrow \tau x$ and set $\lambda=\left(\lambda^{\prime}-1\right) \tau^{m}$. (We take $\lambda=\left(\lambda^{\prime}+1\right) \tau^{m}$ if $P_{m}$ is negative.) Let

$$
H\left(x, h D ; \lambda^{\prime}, h\right)=-h^{2} \Delta+\left(P(x, h)-\lambda^{\prime}\right)^{2},
$$

where $h=\frac{1}{\tau^{m+1}}$ and

$$
P(x, h)=\left(P_{m}(x)+1\right)+h^{1 /(m+1)} P_{m-1}(x)+h^{2 /(m+1)} P_{m-2}(x)+\cdots+h^{m /(m+1)} P_{0} .
$$

Then, the initial problem

$$
H(x, D ; \lambda) v=0
$$

has a nontrivial solution $(\lambda, v)$ if and only if the problem

$$
H\left(x, h D ; \lambda^{\prime}, h\right) u=0
$$

has a nontrivial solution $\left(\lambda^{\prime}, u\right)$ for some $h>0$. We observe that

$$
-h^{2} \Delta+\left(P_{m}+1\right)^{2} \geq 1 \text {. }
$$

It can be proved that if $h>0$ is sufficiently small, then $-h^{2} \Delta+P(x, h)^{2} \geq 1 / 2$, and therefore the latter operator is invertible.

More generally, consider the nonlinear eigenvalue problem

$$
\left(-h^{2} \Delta+(Q(x, h))^{2}+(P(x, h)-z)^{2}\right) u=0,
$$

where $P(x, h)$ is of the above form and

$$
Q(x, h)=Q_{m}(x)+h^{1 /(m+1)} Q_{m-1}(x)+h^{2 /(m+1)} Q_{m-2}(x)+\cdots+h^{m /(m+1)} Q_{0},
$$

with $Q_{j}$ a homogeneous polynomial of degree $j$. This kind of operators arise in the study of nonanalytic hypoellipticity for operators of the form (1.3). We assume that $P_{m}$ and $Q_{m}$ are real and there exists $C>0$ such that

$$
C^{-1}\langle x\rangle^{2 m} \leq\left(P_{m}(x)+1\right)^{2}+\left(Q_{m}(x)\right)^{2} \leq C\langle x\rangle^{2 m}, \quad x \in \mathbb{R}^{n} .
$$

Here $\langle x\rangle=\left(1+|x|^{2}\right)^{1 / 2}$. In the case where $Q_{m}=0, P_{m}$ must be an elliptic polynomial of degree $m$, so that (3.9) be satisfied. By the argument used above, we can show that $-h^{2} \Delta+P(x, h)^{2}+Q(x, h)^{2}$ is invertible for $h>0$ small. In this new setting, we define operators $A=A(h)$ and $B=B(h)$ by the formulas

$$
A(h)=\left(-h^{2} \Delta+P(x, h)^{2}+Q(x, h)^{2}\right)^{-1}, \quad B(h)=A(h)^{1 / 2} P(x, h) A(h)^{1 / 2} .
$$


For a temperate (possibly, $h$-dependent) symbol $K(x, \xi, h)$, we denote by $K(x, h D, h)$ the $h$-pseudodifferential operators defined by Weyl quantization:

$$
K(x, h D, h) u(x)=\frac{1}{(2 \pi h)^{n}} \iint e^{i(x-y) \cdot \xi / h} K\left(\frac{x+y}{2}, \xi, h\right) u(y) d y d \xi,
$$

$u \in \mathcal{S}\left(\mathbb{R}^{n}\right)$. Using the $h$-pseudodifferential calculus (see [18]), we deduce easily that $A(h)$ and $B(h)$ are $h$-pseudodifferential operators with symbols satisfying

$$
\begin{aligned}
& a(x, \xi ; h)=\sum_{j=0}^{(m+1) N} h^{\frac{j}{(m+1)}} a_{j}(x, \xi)+h^{N+\frac{1}{(m+1)}} R_{N}(a, h), \\
& b(x, \xi ; h)=\sum_{j=0}^{(m+1) N} h^{\frac{j}{(m+1)}} b_{j}(x, \xi)+h^{N+\frac{1}{(m+1)}} R_{N}(b, h)
\end{aligned}
$$

for any $N \in \mathbb{N}^{*}$. Here, by definition, the symbols

$$
\begin{aligned}
& a_{0}=\left(\xi^{2}+\left(P_{m}+1\right)^{2}+Q_{m}(x)^{2}\right)^{-1}, \\
& b_{0}=\left(\xi^{2}+\left(P_{m}+1\right)^{2}+Q_{m}(x)^{2}\right)^{-1}\left(P_{m}+1\right)
\end{aligned}
$$

are the $h$-principal symbols of $A(h)$ and $B(h)$, respectively. If we denote by $S_{\phi, \varphi}^{\rho}$ the class of symbols defined as in Robert's book [18], and introduce

$$
\begin{aligned}
\rho_{j} & =\left(1+\xi^{2}+x^{2 m}\right)^{-1}\left(1+x^{2}\right)^{-\frac{j}{2(m+1)}}\left(1+\xi^{2}\right)^{-\frac{j}{2(m+1)}}, \\
\phi & =\left(1+x^{2}\right)^{1 / 2} \\
\varphi & =\left(1+\xi^{2}\right)^{1 / 2}
\end{aligned}
$$

then

$$
a_{j} \in S_{\phi, \varphi}^{\rho_{j}}, \quad b_{j} \in S_{\phi, \varphi}^{\rho_{j}\langle x\rangle^{m}} .
$$

Moreover, the remainders $R_{N}(a, h)$ and $R_{N}(b, h)$ are bounded families of symbols in $S_{\phi, \varphi}^{\rho_{N(m+1)+1}}$ and in $S_{\phi, \varphi}^{\rho_{N(m+1)+1}\langle x\rangle^{m}}$, respectively.

Now, the $k$-criterion of Chanillo, Helffer, and Laptev takes the following form.

Lemma 3.1. Let $\mathcal{D}(h)$ be defined as in (2.6) with $A$ and $B$ replaced by $A(h)$ and $B(h)$. Assume that there exists $k$ such that $\mathcal{D}(h)^{k}$ is an operator of trace class and

$$
\operatorname{Tr} \mathcal{D}(h)^{k} \neq 0
$$

for some $h>0$. Then the nonlinear spectral problem (3.7) has at least one nontrivial solution.

To apply this lemma, we prove the following fact.

Theorem 3.2. Assume that condition (3.9) is fulfilled for $P$ and $Q$ with $m \geq 1$. Let $k>(m+1) / m, n \geq 1$. Then $\mathcal{D}(h)^{k}$ is an operator of trace class for all $h>0$ sufficiently small. For any $N$, we have the following asymptotic expansion:

$$
\operatorname{Tr} \mathcal{D}(h)^{k}=(2 \pi h)^{-n}\left\{\sum_{j=0}^{N(m+1)} h^{\frac{j}{(m+1)}} H_{j ; n, k}+\mathcal{O}\left(h^{N+\frac{1}{(m+1)}}\right)\right\}
$$

as $h \rightarrow 0$. Here the operator $H_{j ; n, k}$ is independent of $h$ and $N$, and can be computed starting with the symbol of $\mathcal{D}(h)^{k}$. In particular,

$$
H_{0 ; n, k}=\int_{\mathbb{R}^{2 n}} \operatorname{tr}\left(\sigma_{k}(x, \xi)\right) d x d \xi,
$$

where $\sigma_{k}$ is the h-principal symbol of $\mathcal{D}(h)^{k}$. 
Proof. We note that $A(h)^{1 / 2}$ and $B(h)$ are $h$-pseudodifferential operators with symbol in $S_{\phi, \varphi}^{\rho_{0}^{1 / 2}}$. Therefore, $\mathcal{D}(h)^{k}$ is an $h$-pseudodifferential operator with matrix-valued symbol of class $S_{\phi, \varphi}^{\rho_{0}^{k / 2}}$. Since $m \geq 1$, we have

$$
\rho_{0}^{k / 2} \in L^{1}\left(\mathbb{R}^{2 n}\right) \text { if } k>n(m+1) / m .
$$

Consequently, $\mathcal{D}(h)^{k}$ is a trace class operator if $k>n(m+1) / m$. Let $\sigma_{k}(x, \xi ; h)$ denote the total symbol of $\mathcal{D}(h)^{k}$. Like $a(h)$, it has a complete semiclassical expansion beginning with $\sigma_{k}$, the $h$-principal symbol of $\mathcal{D}(h)^{k}$. The semiclassical expansion of the trace follows from the formula

$$
\operatorname{Tr} \mathcal{D}(h)^{k}=(2 \pi h)^{-n} \iint \operatorname{tr}\left(\sigma_{k}(x, \xi ; h)\right) d x d \xi .
$$

Here $\operatorname{tr}$ denotes the trace of $(2 \times 2)$-matrices.

We present a consequence of Lemma 3.1 and Theorem 3.2

Corollary 3.3 (The semiclassical criterion). Let (3.2) be satisfied. If there exists $k \in \mathbb{N}$ with $k>(m+1) n / m$ such that $H_{j ; n, k} \neq 0$ for some $j \in \mathbb{N}$, then the nonlinear eigenvalue problem (3.7) has at least one nontrivial solution $(z, u)$ for each $h>0$ sufficiently small.

\section{§4. An Application of the SEMiClassicAl CRITERION: THE ClASSiCAL CRITERION}

In this section, we apply the semiclassical criterion at the classical level, that is, for $j=0$.

Proposition 4.1. Assume that $Q_{m}=0$ and $P_{m} \geq 0$ is elliptic. Let $k>(m+1) n / m$. Then

$$
\begin{array}{ll}
H_{0 ; n, k}=0 & \text { if } n \text { is odd, } \\
H_{0 ; n, k}=2(-1)^{\ell} C_{n} \frac{(n-1) !}{(k-1)(k-2) \cdots(k-n)} C\left(P_{m}\right) & \text { if } n=2 \ell .
\end{array}
$$

Here $C_{n}$ is the volume of $\mathbb{S}^{n-1}$, and

$$
C\left(P_{m}\right)=\int_{\mathbb{R}_{x}^{n}}\left(P_{m}(x)+1\right)^{n-k} d x>0 .
$$

In particular, we observe that

$$
H_{0 ; n, k} \neq 0 \quad \text { if } k>(m+1) n / m
$$

for all $n$ even. As a consequence, we get Theorem 1.1

Proof of Proposition 4.1. Condition (3.9) is satisfied. We compute

$$
\iint \operatorname{tr}\left(\sigma_{k}(x, \xi)\right) d x d \xi
$$

where $\sigma_{k}$ is the $h$-principal symbol of $\mathcal{D}(h)^{k}$. Although the symbolic calculus for matrixvalued $h$-pseudodifferential operators is complicated, the $h$-principal symbol of $\mathcal{D}(h)^{k}$ can easily be computed. Since the $h$-principal symbol of $\mathcal{D}(h)$ is

$$
\left(\begin{array}{cc}
2 b_{0} & a_{0}^{1 / 2} \\
-a_{0}^{1 / 2} & 0
\end{array}\right)
$$

the $h$-principal symbol of $\mathcal{D}(h)^{k}$ is

$$
\sigma_{k}=\left(\begin{array}{cc}
2 b_{0} & a_{0}^{1 / 2} \\
-a_{0}^{1 / 2} & 0
\end{array}\right)^{k}
$$


Therefore,

$$
\operatorname{tr} \sigma_{k}=\left(b_{0}+\sqrt{b_{0}^{2}-a_{0}}\right)^{k}+\left(b_{0}-\sqrt{b_{0}^{2}-a_{0}}\right)^{k} .
$$

We recall that

$$
a_{0}=\left(\xi^{2}+\left(P_{m}+1\right)^{2}\right)^{-1}, \quad b_{0}=\left(\xi^{2}+\left(P_{m}+1\right)^{2}\right)^{-1}\left(P_{m}+1\right) .
$$

By the change of variables $\xi \mapsto\left(P_{m}+1\right) \eta$, we obtain the formula

$$
\iint \operatorname{tr}\left(\sigma_{k}(x, \xi)\right) d x d \xi=C\left(P_{m}\right) \int_{\mathbb{R}_{\eta}^{n}}\left(1+\eta^{2}\right)^{-k}\left((1+i|\eta|)^{k}+(1-i|\eta|)^{k}\right) d \eta .
$$

This shows that

$$
H_{0 ; n, k}=2 C_{n} C\left(P_{m}\right) \operatorname{Re} \int_{0}^{\infty}\left(1+r^{2}\right)^{-k}(1+i r)^{k} r^{n-1} d r, \quad k>(m+1) n / m,
$$

where $C_{n}$ is the volume of $\mathbb{S}^{n-1}$.

We put

$$
L(n, k)=\int_{0}^{\infty}\left(1+r^{2}\right)^{-k}(1+i r)^{k} r^{n-1} d r=\int_{0}^{\infty}(1-i r)^{-k} r^{n-1} d r .
$$

Integration by parts gives

$$
\begin{aligned}
L(n, k) & =i \frac{n-1}{k-1} L(n-1, k-1) \\
& =i^{n-2} \frac{(n-1)(n-2) \cdots 2}{(k-1)(k-2) \cdots(k-n+2)} L(2, k-n+2) .
\end{aligned}
$$

Since

we have

$$
L(2, j)=-\frac{1}{(j-1)(j-2)},
$$

$$
H_{0 ; n, k}=2 C_{n} \operatorname{Re}\left\{i^{n} \frac{(n-1) !}{(k-1)(k-2) \cdots(k-n+1)(k-n)}\right\} C\left(P_{m}\right) .
$$

This proves (4.1) and (4.2).

Using the classical criterion, we can construct a family of examples for $n$ odd.

Corollary 4.2. Let $n=n_{1}+n_{2}$ with $n_{1}$ even and $n_{2} \geq 1$. Let $P$ and $R$ be real elliptic homogeneous polynomial $\mathbb{1}$ of degree $m$ on $\mathbb{R}^{n_{1}}$ and $\mathbb{R}^{n_{2}}$, respectively. For $x \in \mathbb{R}^{n}$, set $x=\left(x^{\prime}, x^{\prime \prime}\right) \in \mathbb{R}^{n_{1}} \times \mathbb{R}^{n_{2}}$. Then the operator

$$
-\Delta_{x}+\left(R\left(x^{\prime \prime}\right) D_{y_{1}}\right)^{2}+\left(P\left(x^{\prime}\right) D_{y_{1}}-D_{y_{2}}\right)^{2}
$$

is not analytic hypoelliptic at 0 in $\mathbb{R}^{n+2}$.

Proof. It suffices to show that the nonlinear eigenvalue problem

$$
\left(\sum_{j=1}^{n} D_{x_{j}}^{2}+\left(R\left(x^{\prime \prime}\right)\right)^{2}+\left(P\left(x^{\prime}\right)-z\right)^{2}\right) u=0
$$

has a nontrivial solution $(z, u)$. We can look for $v\left(x^{\prime}\right)$ satisfying

$$
\left(-\Delta_{x^{\prime}}+\lambda_{0}+\left(P\left(x^{\prime}\right)-z\right)^{2}\right) v=0
$$

for some $z$, where $\lambda_{0}>0$ is an eigenvalue of $-\Delta_{x^{\prime \prime}}+R\left(x^{\prime \prime}\right)^{2}$. The corresponding semiclassical operator is $-h^{2} \Delta_{x^{\prime}}+\lambda_{0} h^{2 m /(m+1)}+\left(P\left(x^{\prime}\right)-z\right)^{2}$ on $\mathbb{R}^{n_{1}}$. Now, we can apply Proposition 4.1 for $Q=Q_{0}=\lambda_{0}$ to complete the proof.

\footnotetext{
${ }^{1}$ The only important property is that the operator $-\Delta_{x^{\prime \prime}}+R\left(x^{\prime \prime}\right)^{2}$ on $\mathbb{R}^{n_{2}}$ has an eigenvalue.
} 
Remark 4.3. We can play with $\lambda_{0}$. In fact, we can indeed consider a sequence $\lambda_{n}$ of eigenvalues of $-\Delta_{x^{\prime \prime}}+R\left(x^{\prime \prime}\right)^{2}$ tending to $+\infty$ and to choose a corresponding sequence $h_{n}$ of $h$ 's tending to 0 and such that $\lambda_{n} h_{n}^{\frac{2 m}{m+1}}=1$. This could permit us to relax the ellipticity condition for $P$, particularly if we add a $Q$ like in (3.7).

Now, we consider the more general case of $Q_{m} \neq 0$. For $\left(P_{m}, Q_{m}\right)$ satisfying (3.9), we can check that the classical criterion still works in some cases. We observe that now

$$
\operatorname{tr} \sigma_{k}=\left(b_{0}+\sqrt{b_{0}^{2}-a_{0}}\right)^{k}+\left(b_{0}-\sqrt{b_{0}^{2}-a_{0}}\right)^{k}
$$

with

$$
a_{0}=\left(\xi^{2}+\left(P_{m}+1\right)^{2}+Q_{m}^{2}\right)^{-1}, \quad b_{0}=\left(\xi^{2}+\left(P_{m}+1\right)^{2}+Q_{m}^{2}\right)^{-1}\left(P_{m}+1\right) .
$$

Let

$$
T=\left(\left(P_{m}+1\right)^{2}+Q_{m}^{2}\right)^{1 / 2}, \quad \tau_{1}=\left(P_{m}+1\right) / T, \quad \tau_{2}=\left|Q_{m}\right| / T .
$$

The change of variables $\xi \rightarrow T \eta$ yields

$$
\int \operatorname{tr}\left(\sigma_{k}\right)(x, \xi) d \xi=2 T^{n-k} \operatorname{Re} \int_{\mathbb{R}_{\eta}^{n}}\left(1+\eta^{2}\right)^{-k}\left(\tau_{1}+i\left(\tau_{2}^{2}+\eta^{2}\right)^{1 / 2}\right)^{k} d \eta
$$

The case of $n=1$. This case can also be analyzed thoroughly by the method of [2]. If $n=1$, for $k=3$ we obtain

$$
H_{0 ; 1,3}=-3 \pi \int_{\mathbb{R}_{x}} \tau_{1} \tau_{2}^{2} T^{-2} d x
$$

It is clear that if $P_{m} \geq 0$ (this requires $m$ to be even) and $Q_{m} \neq 0$, then $\tau_{1} \geq 0$ and $H_{0: 1,3}<0$. If $P_{m}$ changes the sign, $H_{0 ; 1,3}$ may vanish.

The case of $n \geq 2$. If $n \geq 2$ and $k>(m+1) n / m$, then

$$
H_{0 ; n, k}=2 C_{n} \operatorname{Re} \int_{\mathbb{R}_{x}^{n}} T(x)^{n-k} \int_{0}^{\infty}\left(1+r^{2}\right)^{-k}\left(\tau_{1}+i\left(\tau_{2}^{2}+r^{2}\right)^{1 / 2}\right)^{k} r^{n-1} d r d x .
$$

Set

$$
C_{n, k}=\int_{0}^{\infty}\left(1+r^{2}\right)^{-k}\left(\tau_{1}+i\left(\tau_{2}^{2}+r^{2}\right)^{1 / 2}\right)^{k} r^{n-1} d r
$$

By a change of variable, we obtain

$$
C_{n, k}=\int_{\tau_{2}}^{\infty}\left(\tau_{1}-i t\right)^{-k}\left(t^{2}-\tau_{2}^{2}\right)^{(n-2) / 2} t d t
$$

The subcase where $n=2$. For $n=2$, it is easily seen that

$$
C_{2, k}=-\frac{\left(\tau_{1}+i \tau_{2}\right)^{k-2}}{k-2}+\frac{\tau_{1}\left(\tau_{1}+i \tau_{2}\right)^{k-1}}{k-1} .
$$

Since $\tau_{1}^{2}+\tau_{2}^{2}=1$, we can show that

$$
\begin{aligned}
& \operatorname{Re} C_{2,3}=-\tau_{1}\left(1+2 \tau_{2}^{2}\right) / 2 \leq 0 \quad \text { if } \tau_{1} \geq 0 ; \\
& \operatorname{Re} C_{2,4}=\frac{1}{2}-2 \tau_{1}^{2}+\frac{4}{3} \tau_{1}^{4} .
\end{aligned}
$$

Consequently, $H_{0 ; 2,3}<0$ for $n=2$ if $P_{m} \geq 0$. The classical criterion can be used in this case for $m \geq 3$ if $n=2$.

To study the case of $n=2$ and $m=2$, we need information on the sign of $H_{0 ; 2,4}$, which depends on the relationship between $P_{2}$ and $Q_{2}$. Note that $\tau_{1}\left(x_{1}, x_{2}\right)^{2} \in[0,1]$. An elementary calculation gives the following statement. 
Lemma 4.4. (1) If $\tau_{1}\left(x_{1}, x_{2}\right)^{2} \geq(3-\sqrt{3}) / 4$ for all $\left(x_{1}, x_{2}\right)$, then $H_{0 ; 2,4}<0$.

(2) If $\tau_{1}\left(x_{1}, x_{2}\right)^{2} \leq(3-\sqrt{3}) / 4$ for all $\left(x_{1}, x_{2}\right)$, then $H_{0 ; 2,4}>0$.

The subcase where $n=3$. If $k$ is odd, the integration over $r$ in (4.14) is elementary though lengthy. Here we work out the case of $n=3, k=5$. The interested reader can check if our classical criterion can be applied in higher dimensions.

By 4.15),

$$
\operatorname{Re} C_{3,5}=\tau_{1} \int_{0}^{\infty}\left(1+r^{2}\right)^{-5}\left\{\left(\tau_{1}^{4}-10 \tau_{1}^{2} \tau_{2}^{2}+5 \tau_{2}^{4}\right)-10\left(\tau_{1}^{2}-\tau_{2}^{2}\right) r^{2}+5 r^{4}\right\} r^{2} d r .
$$

Making use of the relation $\tau_{1}^{2}+\tau_{2}^{2}=1$ and the formulas

$$
\begin{gathered}
\int_{0}^{\infty}\left(1+r^{2}\right)^{-5} r^{2} d r=\int_{0}^{\infty}\left(1+r^{2}\right)^{-5} r^{6} d r=\frac{5 \pi}{256} \\
\int_{0}^{\infty}\left(1+r^{2}\right)^{-5} r^{4} d r=\frac{3 \pi}{256}
\end{gathered}
$$

we obtain

$$
\operatorname{Re} C_{3,5}=\frac{5 \pi}{16} \tau_{2}^{4} \tau_{1}
$$

It follows that

$$
H_{0 ; 3,5}=2 C_{3} \int_{\mathbb{R}_{x}^{3}} \operatorname{Re} C_{3,5} T^{-2} d x=\frac{5 \pi^{2}}{2} \int_{\mathbb{R}_{x}^{3}} \tau_{2}^{4} \tau_{1} T^{-2} d x>0
$$

whenever $Q_{m}$ is not identically zero and $P_{m} \geq 0$. The classical criterion can be applied for all $m \geq 2$ and $n=3$.

\subsection{Some applications.}

Proposition 4.5. (a) Let $n=1$. The operator

$$
P_{m}(x, D):=D_{x}^{2}+\left(D_{s}-c x^{m} D_{t}\right)^{2}+\left(x^{m} D_{t}\right)^{2}
$$

is not analytic hypoelliptic at $0 \in \mathbb{R}^{3}$ for any even $m \geq 2$ and any $c \in \mathbb{R}$.

(b) Let $n=2$, and let $P$ and $Q$ be real homogeneous polynomials of degree $m \geq 2$ and satisfying (3.9). Suppose $P \geq 0$. If $m=2$, we assume additionally that one of the above conditions on $\tau_{1}$ is satisfied. Then the operator

$$
D_{x}^{2}+D_{y}^{2}+\left(D_{s}-P(x, y) D_{t}\right)^{2}+\left(Q(x, y) D_{t}\right)^{2}, \quad(x, y) \in \mathbb{R}^{3},
$$

is not analytic hypoelliptic at 0 in $\mathbb{R}^{4}$.

(c) Let $n=3$, and let $P$ and $Q$ be real particular polynomials on $\mathbb{R}^{3}$ of degree $m \geq 2$ and satisfying (3.9). Suppose $Q \neq 0, P \geq 0$. Then the operator

$$
-\Delta_{x}+\left(D_{s}-P(x) D_{t}\right)^{2}+\left(Q(x) D_{t}\right)^{2}
$$

is not analytic hypoelliptic at 0 in $\mathbb{R}^{5}$.

We believe that the condition on $\tau_{1}$ in (b) is technical. As examples of $(P, Q)$ satisfying the conditions of (b) in Proposition 4.5, we can take $P(x, y)=\left(x^{2}+y^{2}\right)^{\ell}, Q(x, y)=(x y)^{\ell}$, where $\ell \geq 1$ is arbitrary, because in the case of $\ell=1$ we can check the inequality $\tau_{1}(x, y)^{2} \geq 4 / 5>(3-\sqrt{3}) / 4$. We shall come back to the analysis of this example corresponding to $\ell=1$ in the next section.

Remark 4.6. We briefly compare the results obtained here and those of [2]. Apparently, in the case of $Q=0$, the "classical" criterion does not produce any result for $n \geq 1$ odd. 
But the semiclassical criteria may still work if we consider a coefficient of higher order of the expansion of the trace.

As indicated by J. Sjöstrand, a similar approach was used by L. Nedelec in [16] for getting a lower bound for the number of resonances of an $h$-pseudodifferential system. The same condition on the dimension arises.

The "quantum" criterion given in [2] works for $n=1,2,3$, but with a stronger condition on $m$ for $n>1$. Moreover, in Remark 4.4 in [2] it was observed that the condition of ellipticity of $P$ can be relaxed. The last point is that the homogeneity of $P$ plays an important role in the dilation argument of [2, while under the semiclassical approach the lower order parts can be included. This appears to be useful in the dimension reduction.

\section{§5. Comparison with MÉtivier's Results}

In this section, we would like to analyze the links between our results and the previous work by G. Métivier [12, 14, 15.

5.1. The first family of examples. We start with the following operator on $\mathbb{R}^{n+2}$ :

$$
H\left(X, D_{X}\right)=-\Delta+\left(P(x) D_{x_{n+1}}-D_{x_{n+2}}\right)^{2},
$$

where $X=\left(x, x_{n+1}, x_{n+2}\right)$, and $P$ is a homogeneous positive elliptic polynomial of degree $m \geq 2$ on $\mathbb{R}^{n}$.

If we take the "microlocal spirit", we observe that $H$ is an operator with double characteristics, whose principal symbol is the function

$$
\left(T^{*} \mathbb{R}^{n+2} \backslash 0\right) \ni(X, \Xi) \mapsto|\xi|^{2}+\left(P(x) \xi_{n+1}-\xi_{n+2}\right)^{2} .
$$

This symbol vanishes exactly at order 2 on the submanifold

$$
\Sigma=\left\{(X, \Xi) \mid \xi=0, P(x) \xi_{n+1}-\xi_{n+2}=0, \xi_{n+1} \neq 0\right\} .
$$

This submanifold is of codimension $n+1$. Now, we analyze the "symplecticity" of $\Sigma$. We recall that $\Sigma$ is said to be symplectic if the restriction of the canonical 2-form to $\Sigma$ is nondegenerate. An easy way to verify symplecticity is to consider the $((n+1) \times(n+1))$ matrix $\left\{u_{i}, u_{j}\right\}\left(\{\cdot, \cdot\}\right.$ is the Poisson bracket), where $u_{i}(X, \Xi)=\xi_{i}$ for $i=1, \ldots, n$ and $u_{n+1}(X, \Xi)=P(x) \xi_{n+1}-\xi_{n+2}$, and to show that this matrix is nonsingular. An immediate computation shows that its rank at a given point is 2 if $\nabla P \neq 0$ and 0 if $\nabla P=0$. If $P$ is elliptic and homogeneous, we see that the rank is constant outside 0 and is equal to 2 . There are two cases:

(1) For $n=1$, we see that $\Sigma$ is symplectic except at the points of $\Sigma$ such that $x=0$. The results of Trèves, Tartakoff, Métivier, and Sjöstrand (see [21, 22, 13, 20, 17) shows that the operator in question is microlocally analytic hypoelliptic outside $\Sigma$ (ellipticity) and in the neighborhood of the points of $\Sigma$ such that $x \neq 0$. In this case, the operator is not analytic hypoelliptic at any point $\left(0, x_{n+1}, x_{n+2}\right)$.

(2) For $n>1$, Métivier's result2 [12, 14] implies that the operator $H$ is not analytic hypoelliptic in any open set in $\mathbb{R}^{n+2}$. What we show here is the sharper result that $P$ is not analytic hypoelliptic at any point $\left(0, x_{n+1}, x_{n+2}\right)$, which is a finer property. See the Introduction in [14] for comparison of the definitions of analytic hypoellipticity and germ-hypoanalyticity (analytic hypoellipticity in a neighborhood of a point and analytic hypoellipticity at a point).

5.2. A new class of operators that fail to be analytic hypoelliptic. Now we show that, perhaps, more interesting examples can be treated if we consider the more

\footnotetext{
${ }^{2}$ Note that the operator is hypoelliptic with the loss of one derivative in $\mathbb{R}^{n+2} \backslash\{x=0\}$.
} 
general class:

$$
H\left(X, D_{X}\right)=-\Delta+\left(P(x) D_{x_{n+1}}-D_{x_{n+2}}\right)^{2}+Q(x)^{2} D_{x_{n+1}}^{2}
$$

where $P$ and $Q$ are homogeneous polynomials of degree $m>1$ with $P \geq 0$ and $P^{2}+$ $Q^{2}$ elliptic. When restricting $H$ to $x_{n+2}$-independent distributions, we get an analytic hypoelliptic operator on $\mathbb{R}^{n+1}$, namely,

$$
-\Delta_{x}+\left(P(x)^{2}+Q(x)^{2}\right) D_{x_{n+1}}^{2}
$$

this follows from a theorem of Grushin 8 .

In 4 we saw that the "classical" criterion may give a result under some additional condition. We shall focus our analysis on the specific case where

$$
n=2, \quad P(x)=x_{1}^{2}+x_{2}^{2}, \quad Q(x)=\alpha x_{1} x_{2}, \quad \alpha>0 .
$$

We do the same microlocal analysis as in the preceding subsection. Now the characteristic set $\Sigma$ is defined as the union of two regular submanifolds of dimension 4 in $\mathbb{R}^{8} \backslash 0$ :

$$
\begin{gathered}
\Sigma=\Sigma_{1} \cup \Sigma_{2}, \\
\Sigma_{j}=\left\{\xi_{1}=0, \xi_{2}=0, \xi_{4}=\left(x_{1}^{2}+x_{2}^{2}\right) \xi_{3}, x_{j}=0, \xi_{3} \neq 0\right\} .
\end{gathered}
$$

Moreover, $\Sigma_{j}$ is symplectic outside $\Sigma_{1} \cap \Sigma_{2}$ and is not symplectic at $\Sigma_{1} \cap \Sigma_{2}$.

Outside $\Sigma_{1} \cap \Sigma_{2}$, the symbol of $H$ vanishes exactly at order 2 on $\Sigma$; so, again from [21, $22,13,20$. we deduce that $H$ is microlocally analytic hypoelliptic.

Métivier's criterion of nonanalytic hypoellipticity cannot be applied at the points $\left(0,0, x_{3}, x_{4}\right)$ (the operator is indeed not hypoelliptic with loss of one derivative), and it is of interest to see what is obtained through our approach.

Proposition 5.1. For $\alpha>0$ sufficiently small, the operator $H\left(X, D_{X}\right)$ is not analytic hypoelliptic at any point $\left(0, x_{3}, x_{4}\right)$.

Proof. We apply the criterion of the preceding section (Proposition 4.5, the second case) and the discussion following the statement.

If $\alpha$ is small enough (at least $0<\alpha \leq 1)$, then $\left(P_{m}+1\right)^{2} /\left(\left(P_{m}+1\right)^{2}+Q_{m}^{2}\right)$ is sufficiently close to 1 . In particular, the second condition on $\tau_{1}$ in Lemma 4.4 is satisfied.

Remark 5.2. As was explained to us by M. Christ, for rather large classes of models depending analytically on an additional parameter $\alpha$, it is possible to prove that some associated Fredholm determinant is analytic in $\alpha$. This implies that, if the operator is not analytic hypoelliptic for some value of $\alpha$, then it is not analytic hypoelliptic for generic values of $\alpha$. We refer to [5, Proposition 5.2] for the argument. In the particular case of the above Proposition 5.1, we can present the following argument. Let $H_{0,2,4}(\alpha)$ denote $H_{0,2,4}$ defined as in Theorem 3.2 with $P=x_{1}^{2}+x_{2}^{2}$ and $Q=\alpha x_{1} x_{2}$. Using (4.12) and (4.16), we check that $H_{0,2,4}(\alpha)$ is real analytic in $\alpha>0$. The proof of Proposition 5.1 shows that $H_{0,2,4}(\alpha) \neq 0$ for $\alpha>0$ small. Thus, $H_{0 ; 2,4}(\alpha) \neq 0$ for all $\alpha>0$ except for a discrete set in $\mathbb{R}_{+}$; therefore, Proposition 5.1 remains true in this case. This argument can be used in more general cases. In many situations, it is indeed easy to check the analyticity of $H_{0, n, k}$ with respect to the parameter.

\section{REFERENCES}

[1] S. Chanillo, Kirillov theory, Trèves strata, Schrödinger equations and analytic hypoellipticity of sums of squares, Preprint, August 2001; http://arxiv.org/pdf/math.AP/0107106.

[2] S. Chanillo, B. Helffer, and A. Laptev, Nonlinear eigenvalues and analytic hypoellipticity, Preprint, Inst. Mittag-Leffler; http://arxiv.org/pdf/math.AP/0211308; J. Funct. Anal. 209 (2004), no 2, 425-443. MR2044230

[3] M. Christ, Some nonanalytic-hypoelliptic sums of squares of vector fields, Bull. Amer. Math. Soc. (N.S.) 26 (1992), no. 1, 137-140. MR1110438 (92e:35055) 
[4] — Analytic hypoellipticity, representations of nilpotent groups, and a nonlinear eigenvalue problem, Duke Math. J. 72 (1993), 595-639. MR.1253617|(94k:35075)

[5] _ Analytic hypoellipticity in dimension two, MSRI Preprint no. 009-96, Math. Sci. Res. Inst., Berkeley, CA, 1996.

[6] _ . The Szegö projection need not preserve global analyticity, Ann. of Math. (2) 143 (1996), 301-330. MR 1381988 (97e:32027)

[7] A. Grigis and S. Sjöstrand, Front d'onde analytique et sommes de carrés de champs de vecteurs, Duke Math. J. 52 (1985), no. 1, 35-51. MR0791290 (86h:58136)

[8] V. V. Grushin, A certain class of elliptic pseudodifferential operators that are degenerate on a submanifold, Mat. Sb. (N.S.) 84 (1971), no. 2, 163-195; English transl., Math. USSR-Sb. 13 (1971), 155-185. MR0283630(44:860)

[9] N. Hanges and A. A. Himonas, Nonanalytic hypoellipticity in the presence of symplecticity, Proc. Amer. Math. Soc. 126 (1998), no. 2, 405-409. MR1422872 (98d:35031)

[10] B. Helffer, Conditions nécessaires d'hypoanalyticité pour des opérateurs invariants à gauche homogènes sur un groupe nilpotent gradué, J. Differential Equations 44 (1982), no. 3, 460-481. MR0661164 (84c:35026)

[11] A. S. Markus, Introduction to the spectral theory of polynomial operator pencils, "Shtiintsa", Kishinev, 1986; English transl., Transl. Math. Monogr., vol. 71, Amer. Math. Soc., Providence, RI, 1988. MR0971506 (89h:47023)

[12] G. Métivier, Une classe d'opérateurs non hypoelliptiques analytiques, Séminaire Goulaouic-Schwartz (1978-1979), École Polytech., Palaiseau, 1979, Exp. No. 12. MR0557523 (83f:35039)

[13] __ Analytic hypoellipticity for operators with multiple characteristics, Comm. Partial Differential Equations 6 (1981), 1-90. MR0597752 (82g:35030)

[14] Une classe d'opérateurs non hypoelliptiques analytiques, Indiana Univ. Math. J. 29 (1980), 823-860. MR0589650 (82a:35029)

[15] _ Non hypoellipticité analytique pour des opérateurs à caractéristiques doubles, GoulaouicMeyer-Schwartz Seminar, 1981/1982, École Polytech., Palaiseau, 1982, Exp. No. 12. MR0671609 (84c:35027)

[16] L. Nedelec, Existence of resonances for matrix Schrödinger operators, Asymptot. Anal. 35 (2003), no. 3-4, 301-324. MR2011792 (2004i:35065)

[17] Pham The Lai and D. Robert, Sur un problème aux valeurs propres non linéaire, Israel J. Math. 36 (1980), no. 2, 169-186. MR0623203 (83b:35132)

[18] D. Robert, Autour de l'approximation semi-classique, Progr. Math., vol. 68, Birkhäuser Boston, Inc., Boston, MA, 1987. MR0897108 (89g:81016)

[19] B. Simon, Trace ideals and their applications, London Math. Soc. Lecture Note Ser., vol. 35, Cambridge Univ. Press, Cambridge-New York, 1979. MR0541149 (80k:47048)

[20] J. Sjöstrand, Analytic wavefront sets and operators with multiple characteristics, Hokkaido Math. J. 12 (1983), 392-433. MR0725588 (85e:35022)

[21] D. Tartakoff, The local real analyticity of solutions to $\square b$ and the $\bar{\partial}$-Neumann problem, Acta Math. 145 (1980), 117-204. MR0590289 (81k:35033)

[22] F. Trèves, Analytic hypo-ellipticity of a class of pseudo-differential operators with double characteristics and applications to the $\bar{\partial}$-Neumann problem, Comm. Partial Differential Equations 3 (1978), no. 6-7, 475-642. MR0492802 (58:11867)

[23] _ Symplectic geometry and analytic hypo-ellipticity, Differential Equations: La Pietra 1996 (Florence), Proc. Sympos. Pure Math., vol. 65, Amer. Math. Soc., Providence, RI, 1999, pp. 201219. MR.1662756 (2000b:35031)

Département de Mathématiques, UMR CNRS 8628, Université Paris-Sud, Bat. 425, 91405 Orsay Cedex, France

E-mail address: Bernard.Helffer@math.u-psud.fr

Laboratoire de Mathématiques Jean Leray, Département de Mathématiques, UMR CNRS 6629, Université de Nantes, 44322 Nantes Cedex 3, France

E-mail address: Didier.Robert@math.univ-nantes.fr

Laboratoire de Mathématiques Jean Leray, Département de Mathématiques, UmR CNRS 6629, Université de Nantes, 44322 Nantes Cedex 3, France

E-mail address: Xue-Ping.Wang@math.univ-nantes.fr 